\title{
Threshold and jet radius joint resummation for single-inclusive jet production
}

\author{
Sven-Olaf Moch* \\ II. Institut für Theoretische Physik, Universität Hamburg, Luruper Chaussee 149, D-22761 \\ Hamburg, Germany \\ E-mail: sven-olaf.moch@desy.de
}

\section{Engin Eren}

Deutsches Elektronen Synchrotron DESY, Notkestraße 85, D-22607 Hamburg, Germany

E-mail: engin.eren@desy.de

\section{Katerina Lipka}

Deutsches Elektronen Synchrotron DESY, Notkestraße 85, D-22607 Hamburg, Germany

E-mail: katerina.lipka@desy.de

\section{Xiaohui Liu}

Center of Advanced Quantum Studies, Department of Physics, Beijing Normal University, Beijing 100875, China

E-mail: xiliu@bnu.edu.cn

\section{Felix Ringer}

Nuclear Science Division, Lawrence Berkeley National Laboratory, Berkeley, California 94720 , USA

E-mail: fmringerelbl.gov

The QCD predictions for single-inclusive jet production are computed with joint resummation of threshold and jet radius logarithms. The results are compared to those based on fixed order perturbation theory up to next-to-next-to-leading order and to data by the CMS collaboration measured in proton-proton collisions at the LHC at $\sqrt{S}=8 \mathrm{TeV}$. The joint resummation results are in remarkable agreement with the data.

Loops and Legs in Quantum Field Theory (LL2018)

29 April 2018 - 04 May 2018

St. Goar, Germany

${ }^{*}$ Speaker. 
The hadro-production of jets in proton-proton collisions at the Large Hadron Collider (LHC) is one of the basic processes which exposes all features of the gauge theory of the strong interactions, quantum chromodynamics (QCD). Within standard QCD factorization the description of jet hadro-production starts off at Born level as a hard $2 \rightarrow 2$ scattering reaction of partons in the incident protons and its theory prediction is directly proportional to powers of the strong coupling constant $\alpha_{s}$ and to the parton luminosity, i.e., the distribution of the fractions of parton momenta relative to those of the colliding protons. These characteristics make jet hadro-production a very interesting process for determinations of both, the value of the strong coupling $\alpha_{s}$ as well as the parton distribution functions (PDFs) from collider data, see for instance Refs. [1, 2].

The single-inclusive jet production cross section, i.e., the observable of interest for us here, is obtained by summing over all jets that are observed in an event. The corresponding double differential expression for a jet of cone size $R$ reads

$$
\frac{p_{T}^{2} \mathrm{~d}^{2} \sigma}{\mathrm{d} p_{T}^{2} \mathrm{~d} y}=\sum_{i_{1} i_{2}} \int_{0}^{V(1-W)} \mathrm{d} z \int_{\frac{V W}{1-z}}^{1-\frac{1-V}{1-z}} \mathrm{~d} v x_{1}^{2} f_{i_{1}}\left(x_{1}\right) x_{2}^{2} f_{i_{2}}\left(x_{2}\right) \frac{\mathrm{d}^{2} \hat{\sigma}_{i_{1} i_{2}}}{\mathrm{~d} v \mathrm{~d} z}\left(v, z, p_{T}, R\right),
$$

where $p_{T}$ and $y$ denote the transverse momentum and rapidity of the jet. The sums $i$ run over all partonic channels which contribute through the convolution of the hard partonic cross sections $\hat{\sigma}_{i_{1} i_{2}}$ with PDFs $f_{i}$ evaluated at the fractions $x_{1}=V W / v /(1-z)$ and $x_{2}=(1-V) /(1-v) /(1-z)$ of the proton momenta, where $V=1-p_{T} e^{-y} / \sqrt{S}, V W=p_{T} e^{y} / \sqrt{S}$ and $\sqrt{S}$ is the hadronic center-ofmass energy. The partonic kinematic variables are $s=x_{1} x_{2} S, v=u /(u+t)$ and $z=s_{4} / s$, defined in terms of the standard Mandelstam variables $t, u$ and, respectively, the invariant mass $s_{4}$ of the partonic system recoiling against the observed jet, cf. Ref. [3].

The respective higher order perturbative QCD predictions to the partonic cross sections $\hat{\sigma}_{i_{1} i_{2}}$ in Eq. (1) are known to next-to-leading order (NLO) since long $[4,5]$ while those at next-to-next-toleading order (NNLO) have been computed recently in the leading-color approximation [6], that is for large values of $N_{c}$ for a general $\mathrm{SU}\left(N_{c}\right)$ gauge group with additional corrections parametrically suppressed as $1 / N_{c}^{2}$. This is supposed to approximate the full NNLO calculation very well.

Higher order corrections to hard processes in QCD are generally expected to display an apparent convergence of the perturbative expansion as well as to show a significant reduction of the dependence on the scales $\mu_{R}$ for ultraviolet renormalization and $\mu_{F}$ for QCD factorization. Both these features lead to a stabilization of the theoretical predictions. However, comparisons of the fixed order NNLO results of Ref. [6] for the single-inclusive jet production cross section with some of the LHC data have not been entirely satisfactory [7, 8]. Moreover, the NNLO corrections do change significantly depending on the chosen values for the hard scales $\mu_{R}$ and $\mu_{F}$ (denoted here collectively by $\mu$ ). Choices like $\mu=p_{T}^{\max }$ with the natural hard scale of the transverse momentum $p_{T}^{\max }$ of the leading jet or, alternatively, scales like $\mu=p_{T}$ with the transverse momentum $p_{T}$ of each individual jet in the event lead to completely different theoretical predictions. The latter choice $\mu=p_{T}$ typically involves much softer scales because kinematical configurations in events with three or more hard jets or events with hard emissions outside the jet fiducial cuts such as smaller jet cone radii $R$ generate a hierarchy in the transverse momenta between the leading and subleading jets in the event, $p_{T}^{\max }=p_{T, 1} \gg p_{T, 2} \geq p_{T, 3} \ldots$, cf. Ref. [9].

This infrared sensitivity implies the existence of large higher order corrections beyond fixed order in perturbation theory and merits a short discussion of their origins. Large logarithms in QCD 
perturbation theory arise systematically from the cancellations of infrared divergences between real and virtual corrections near some boundary of the phase space. For single-inclusive jet production this is realized near threshold for large $p_{T}$, when the event kinematics are almost Born-like and double logarithms appear in the partonic cross section at $n$-th order as $\alpha_{s}^{n}\left(\ln ^{k}(z) / z\right)_{+}$where $k \leq$ $2 n-1$ and $z$ is the measure for the distance from partonic threshold, cf. Eq. (1). On the other hand, the definition of jets via their cone sizes $R$ as an external quantity introduces large singlelogarithmic corrections in the partonic cross section at $n$-th order as $\alpha_{s}^{n} \ln ^{k}(R)$ with $k \leq n$.

Both, threshold and small- $R$ logarithms require an all-order resummation and one expects competing effects from these two sources. The resummation of threshold logarithms leads to an enhancement of the cross section for large $p_{T}$ and has been studied in Refs. [10, 11, 12]. On the contrary, the resummation of small- $R$ logarithms alone leads to a decrease of the cross section in the entire range of $p_{T}[13,14]$. A framework for the joint resummation of both, threshold and jet radius logarithms simultaneously, has recently been developed in Ref. [3]. To that end the partonic cross sections $\hat{\sigma}_{i_{1} i_{2}}$ in Eq. (1) are further factorized within the soft collinear effective theory (SCET) [15] as

$$
\begin{aligned}
\frac{\mathrm{d}^{2} \hat{\sigma}_{i_{1} i_{2}}}{\mathrm{~d} v \mathrm{~d} z}=s \int & \mathrm{d} s_{X} \mathrm{~d} s_{c} \mathrm{~d} s_{G} \boldsymbol{\delta}\left(z s-s_{X}-s_{G}-s_{c}\right) \operatorname{Tr}\left[\mathbf{H}_{i_{1} i_{2}}\left(v, p_{T}, \mu_{h}, \mu\right) \mathbf{S}_{G}\left(s_{G}, \mu_{s G}, \mu\right)\right] \\
& \times J_{X}\left(s_{X}, \mu_{X}, \mu\right) \sum_{m} \operatorname{Tr}\left[J_{m}\left(p_{T} R, \mu_{J}, \mu\right) \otimes_{\Omega} S_{c, m}\left(s_{c} R, \mu_{s c}, \mu\right)\right]
\end{aligned}
$$

where the specific functions capture the dynamics of individual kinematic regions, i.e., the function $\mathbf{H}_{i_{1} i_{2}}$ for the underlying hard $2 \rightarrow 2$ scattering, the global soft function $\mathbf{S}_{G}$ for wide angle soft radiation from partons, which cannot resolve the jet with a small radius $R$ and the soft collinear ('coft') function $S_{c}\left(s_{c} R\right)$ for soft radiation near the jet boundary. Likewise, the inclusive jet function $J_{X}\left(s_{X}\right)$ describes the recoiling collimated radiation with invariant mass $s_{X}$, while the signal-jet function $J_{m}\left(p_{T} R\right)$ accounts for energetic radiation inside jet. The sum in Eq. (2) runs over all collinear splittings, the traces are to be taken in color space and ' $\otimes$ ' denotes the associated angular integrals. The functions, $\mathbf{H}_{i_{1} i_{2}}, \mathbf{S}_{G}, J_{X}\left(s_{X}\right), S_{c}\left(s_{c} R\right)$ and $J_{m}\left(p_{T} R\right)$ in Eq. (2) are known to NLO at least, which allows to perform the joint resummation at next-to-leading logarithmic (NLL) accuracy, by evolving all functions with their renormalization group equations from their natural scales $\mu_{i}$ to a common hard scale $\mu=\mu_{h}=p_{T}^{\max }$, see Ref. [3] for details.

The SCET factorization used in Eq. (2) holds in the threshold regime for $z \rightarrow 0$ and for small jet radii, $R \ll 1$ and is matched to the fixed order QCD result as follows,

$$
\sigma_{\mathrm{NLO}+\mathrm{NLL}}=\sigma_{\mathrm{NLO}}-\sigma_{\mathrm{NLO}_{\text {sing }}}+\sigma_{\mathrm{NLL}}
$$

where $\sigma_{\mathrm{NLO}}\left(\sigma_{\mathrm{NLL}}\right)$ denotes the NLO fixed order (NLL resummed) result and $\sigma_{\mathrm{NLO}}$ sing subtracts the logarithmically enhanced contributions at NLO in QCD to avoid double counting.

The first phenomenological studies of the joint jet radius and threshold resummation results with Eq. (3) and comparisons to LHC data have been carried out in Refs. [3, 16] and have shown that the large threshold and the small- $R$ logarithms are indeed responsible for the bulk of the radiative corrections in the kinematic range from moderate to large jet- $p_{T}$. Moreover, the effect of small- $R$ resummation is dominant, even for larger cone sizes, although less pronounced, while threshold logarithms are relevant for very large values of jet- $p_{T}$ and large cone sizes. In particular, 


\begin{tabular}{|c|c|c|c|}
\hline data set & NLO & NNLO & NLO + NLL \\
\hline $0.0 \leq|y|<0.5$ & $36 / 33$ & $55 / 33$ & $39 / 33$ \\
$0.5 \leq|y|<1.0$ & $34 / 32$ & $37 / 32$ & $35 / 32$ \\
$1.0 \leq|y|<1.5$ & $22 / 31$ & $32 / 31$ & $28 / 31$ \\
$1.5 \leq|y|<2.0$ & $10 / 26$ & $26 / 26$ & $12 / 26$ \\
$2.0 \leq|y|<2.5$ & $8 / 19$ & $25 / 19$ & $9 / 19$ \\
$2.5 \leq|y|<3.0$ & $8 / 16$ & $15 / 16$ & $8 / 16$ \\
\hline total $\chi^{2} /$ dof & $142 / 157$ & $229 / 157$ & $154 / 157$ \\
\hline
\end{tabular}

Table 1: The values of $\chi^{2} /$ dof for the QCD theory predictions at NLO, NNLO and NLO + NLL accuracy using the CT14 PDF set at NNLO [18] and the scale choice $\mu_{R}=\mu_{F}=p_{T}^{\max }$ for the single-inclusive jet production cross sections in various rapidity bins measured by CMS at $\sqrt{S}=8 \mathrm{TeV}$ [19].

the use of the resummed prediction $\sigma_{\mathrm{NLO}+\mathrm{NLL}}$ indicates a clear systematic improvement in the description at the available data. It is also worth emphasizing, that in a different framework, threshold resummation for jets including the effects of the jet cone size $R$ has recently been studied in the parton shower event generator Deductor [17] and the findings agree with ours.

Comparisons of jet data collected at the LHC to theory predictions beyond fixed order NLO in QCD are not yet widespread in the literature. This is due to the large computational overhead in the preparation of suitable fast interfaces for such studies, e.g., fast $N L O$ [20]. Here, we continue these phenomenological studies for single-inclusive jet production data measured by CMS at $\sqrt{S}=$ $8 \mathrm{TeV}$ [19] with the anti- $k_{T}$ clustering algorithm [21] for a size parameter of $R=0.7$. The high statistics CMS data for the double-differential inclusive jet cross section in $p_{T}$ and $y$ correspond to an integrated luminosity of 19.7 inverse femtobarn. We confront these data to the QCD theory predictions based on Eq. (1) at NLO, NNLO and NLO + NLL, using Eq. (3) in the latter case. The set-up is as follows. The scales are chosen as $\mu_{R}=\mu_{F}=p_{T}^{\max }$ and in all cases, we employ the CT14 PDF set at NNLO [18] with the value of $\alpha_{s}\left(M_{Z}\right)=0.1180$, independent of the perturbative order.

In Tab. 1 we display the values of $\chi^{2} /$ dof (degree of freedom) for the respective theory predictions for each rapidity bin. The total CMS data set at $\sqrt{S}=8 \mathrm{TeV}$ [19] yields $\chi^{2} / \mathrm{dof}=142 / 157$ when compared to the fixed order NLO prediction, $\chi^{2} / \mathrm{dof}=229 / 157$ at NNLO and $\chi^{2} / \mathrm{dof}=154 / 157$ at NLO + NLL. Thus, the fixed order theory description at NNLO with the hard scale $\mu=p_{T}^{\max }$ is clearly disfavored, an observation in line with the earlier studies of Refs. [7, 8].

In Figs. 1, 2 and 3 we show both, the absolute cross section for each rapidity bin as well as the separate ratios $\sigma_{\text {Data }} / \sigma_{\text {Theory }}$ for the theory predictions at NLO, NNLO and NLO + NLL. While all predictions undershoot the data at the largest values of $p_{T}$, e.g., $p_{T} \gtrsim 1 \mathrm{TeV}$ for central rapidities, their individual trends for moderate values of $p_{T}$ are completely different and the NNLO results overshoot the data significantly. The joint resummation results on the other hand show remarkable agreement. It should be mentioned here, that although the jet cone size of $R=0.7$ is already somewhat larger, the studies of Refs. [3, 16] have shown that there is still significant numerical 

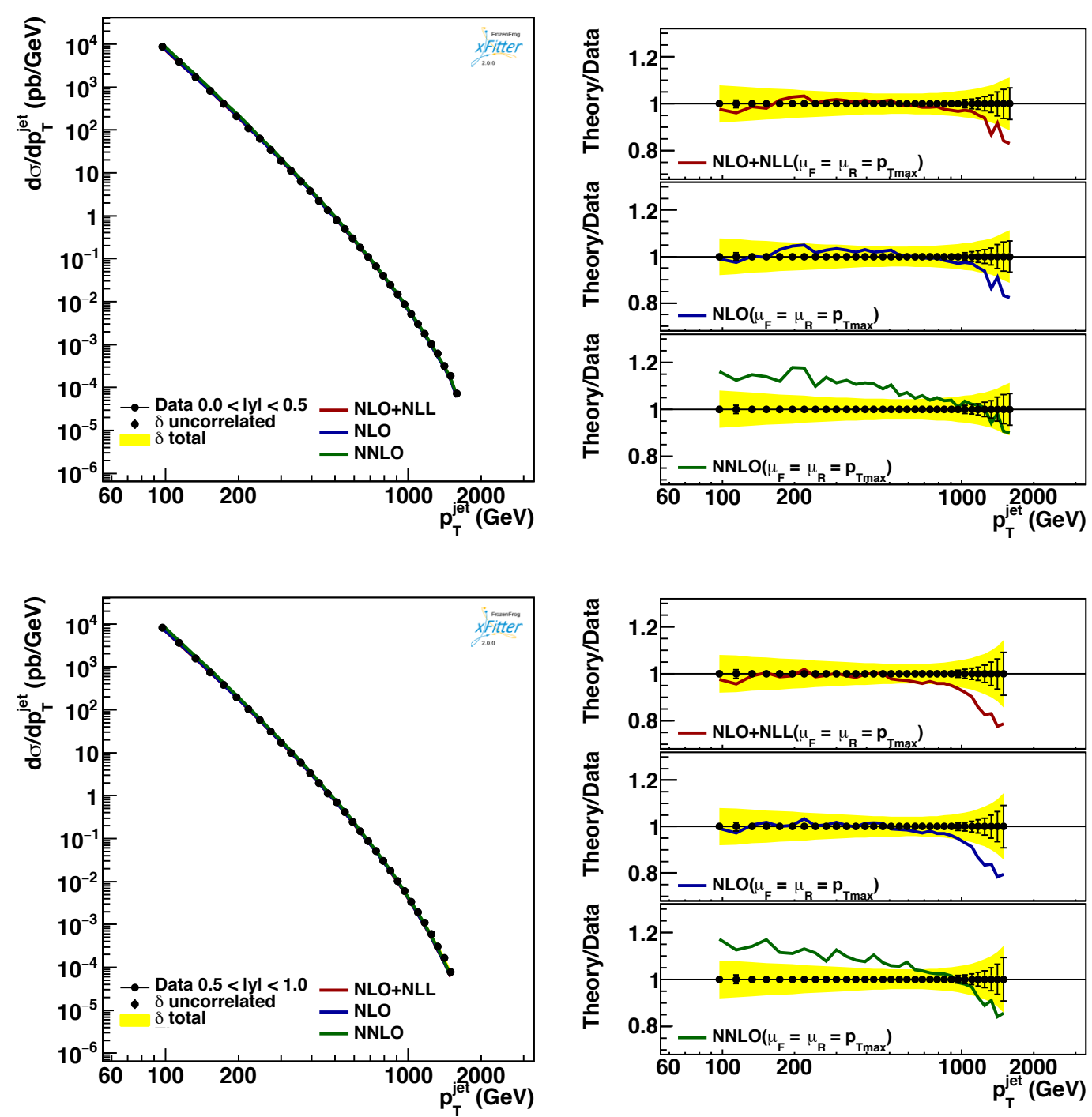

Figure 1: The single-inclusive jet production cross section for $p p \rightarrow$ jet $+\mathrm{X}$ as a function of the jet $p_{T}$ as measured by CMS at $\sqrt{S}=8 \mathrm{TeV}$ [19] for a size parameter of $R=0.7$ in the rapidity bins $0.0 \leq|y|<0.5$ and $0.5 \leq|y|<1.0$ compared to the QCD theory predictions at NLO (blue), NNLO (green) and NLO + NLL (red) accuracy using the CT14 PDF set at NNLO [18] and the scale choice $\mu_{R}=\mu_{F}=p_{T}^{\max }$. The left panel shows the absolute cross section, the right panel the ratio $\sigma_{\text {Data }} / \sigma_{\text {Theory }}$.

impact due to the small- $R$ resummation.

While the Figs. 1, 2 and 3 are based on CT14 PDF set at NNLO [18] the findings concerning the improved quality of the joint resummation result in the description of the data do not significantly depend on the PDF choice, as discussed in Refs. [16, 22]. In particular, these aspects are investigated in detail in a full QCD fit [22], in which both, the PDFs and $\alpha_{s}$ are determined simultaneously using single-inclusive jet data by CMS at $\sqrt{S}=8$ and $13 \mathrm{TeV}$. The conclusion is made, that the deficits of the fixed order NNLO predictions to describe the observed cross sections cannot be mitigated by changes in the PDFs or the strong coupling. 

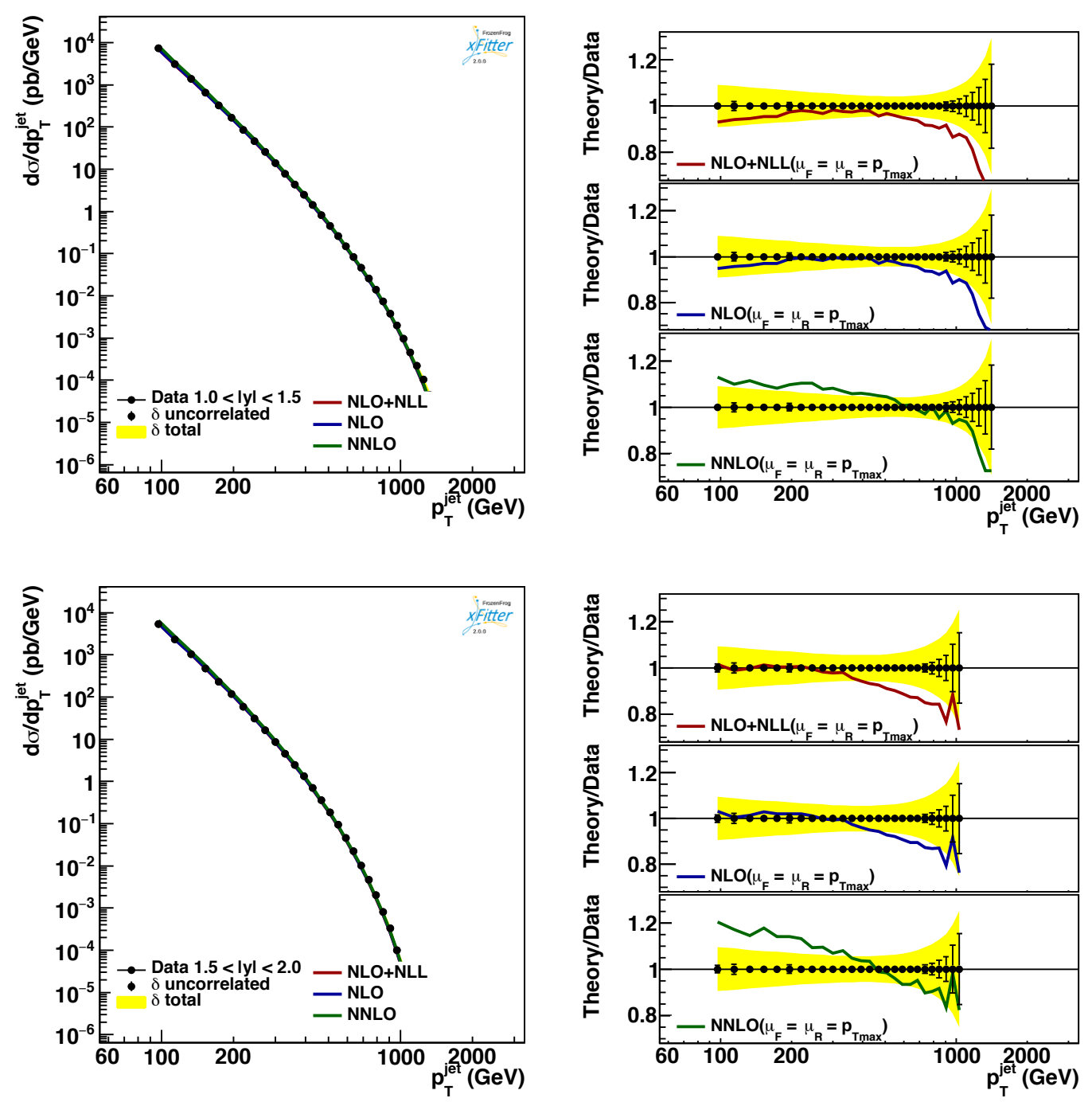

Figure 2: Same as Fig. 1 for the rapidity bins $1.0 \leq|y|<1.5$ and $1.5 \leq|y|<2.0$.

In summary, we note that the NLO + NLL calculations greatly improve the theoretical predictions. However, they do exhibit associated scale uncertainties (not displayed in Figs. 1, 2 and 3), which are still large $[3,16]$ and require improving the joint threshold and small- $R$ joint resummation to next-to-next-to-leading logarithmic (NNLL) accuracy. This can be achieved by computing the individual functions in SCET factorization in Eq. (2) to NNLO so that the susequent evolution with the renormalization group equations then resums all logarithms to NNLL. This will be subject of future work.

\section{Acknowledgments}

The plots have been generated in the $x$ Fitter framework [23, 24].

S.M. acknowledges contract $05 \mathrm{H} 15 \mathrm{GUCC} 1$ by BMBF. X.L. is supported by the National Nat- 

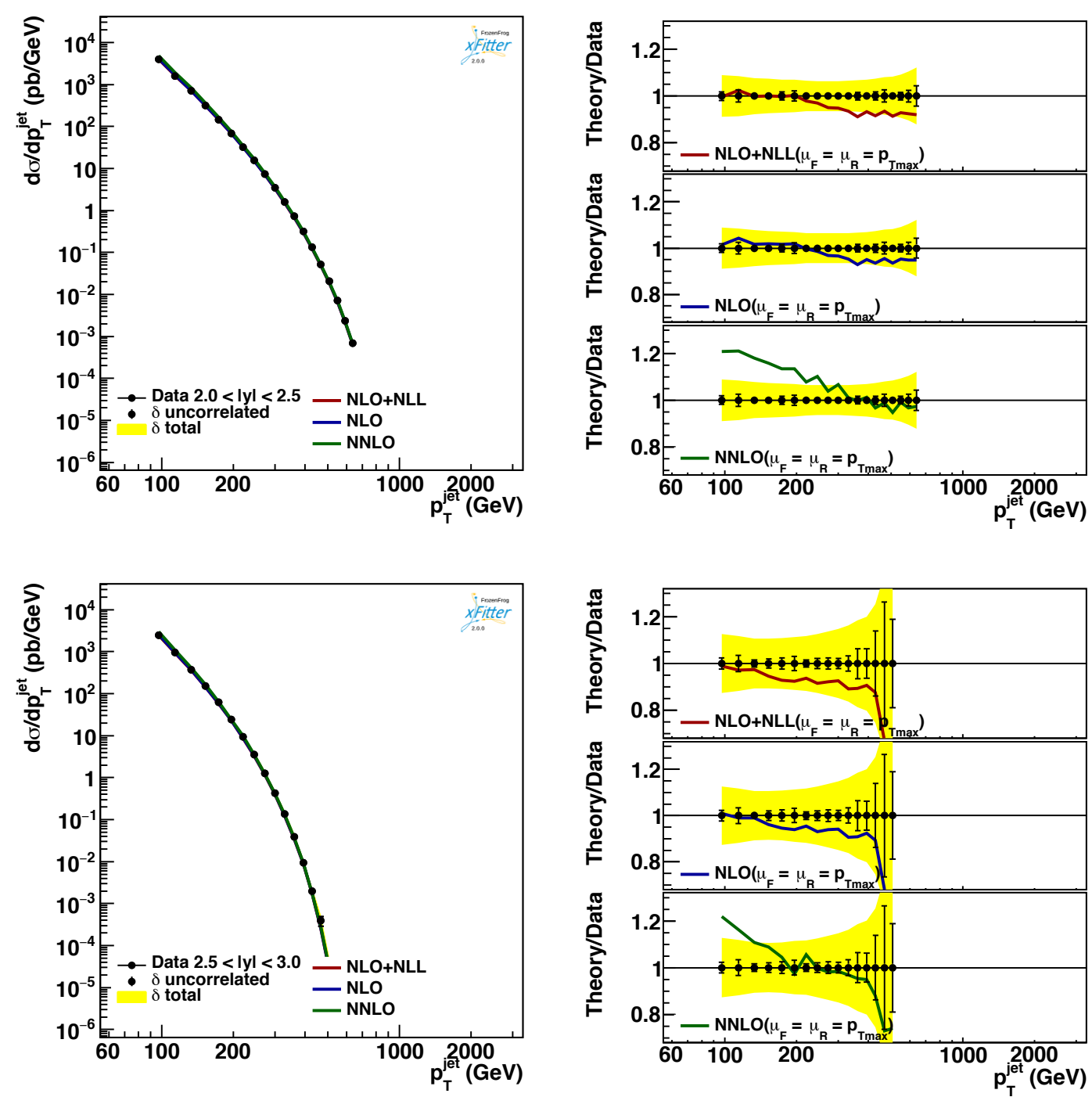

Figure 3: Same as Fig. 1 for the rapidity bins $2.0 \leq|y|<2.5$ and $2.5 \leq|y|<3.0$.

ural Science Foundation of China under Grant No. 11775023 and the Fundamental Research Funds for the Central Universities. F.R. is supported by the Department of Energy under Contract No. DE-AC0205CH11231, and the LDRD Program of LBNL.

\section{References}

[1] A. Accardi et al., A Critical Appraisal and Evaluation of Modern PDFs, Eur. Phys. J. C76 (2016), no. 8 471, [arXiv:1603.08906].

[2] D. Britzger, K. Rabbertz, D. Savoiu, G. Sieber, and M. Wobisch, Determination of the strong coupling constant from inclusive jet cross section data from multiple experiments, arXiv: 1712.00480.

[3] X. Liu, S.-O. Moch, and F. Ringer, Threshold and jet radius joint resummation for single-inclusive jet production, Phys. Rev. Lett. 119 (2017), no. 21 212001, [arXiv: 1708.04641 ]. 
[4] S. D. Ellis, Z. Kunszt, and D. E. Soper, Two jet production in hadron collisions at order $\alpha_{s}^{3}$ in $Q C D$, Phys. Rev. Lett. 69 (1992) 1496-1499.

[5] Z. Nagy, Three jet cross-sections in hadron hadron collisions at next-to-leading order, Phys. Rev. Lett. 88 (2002) 122003, [hep-ph/ 0110315$].$

[6] J. Currie, E. W. N. Glover, and J. Pires, Next-to-next-to leading order QCD predictions for single jet inclusive production at the LHC, Phys. Rev. Lett. 118 (2017), no. 7 072002, [arXiv: 1611.01460 ].

[7] J. Currie, E. W. N. Glover, T. Gehrmann, A. Gehrmann-De Ridder, A. Huss, and J. Pires, Single Jet Inclusive Production for the Individual Jet $p_{\mathrm{T}}$ Scale Choice at the LHC, Acta Phys. Polon. B48 (2017) 955-967, [arXiv:1704.00923].

[8] ATLAS Collaboration, T. A. collaboration, Measurement of inclusive jet and dijet cross-sections in proton-proton collisions at $\sqrt{S}=13 \mathrm{TeV}$ with the ATLAS detector, .

[9] J. Currie, A. Gehrmann-De Ridder, T. Gehrmann, E. W. N. Glover, A. Huss, and J. Pires, Infrared sensitivity of single jet inclusive production at hadron colliders, arXiv : 1807.03692.

[10] N. Kidonakis, G. Oderda, and G. F. Sterman, Threshold resummation for dijet cross-sections, Nucl. Phys. B525 (1998) 299-332, [hep-ph/9801268].

[11] M. C. Kumar and S.-O. Moch, Phenomenology of threshold corrections for inclusive jet production at hadron colliders, Phys. Lett. B730 (2014) 122-129, [arXiv:1309.5311].

[12] D. de Florian, P. Hinderer, A. Mukherjee, F. Ringer, and W. Vogelsang, Approximate next-to-next-to-leading order corrections to hadronic jet production, Phys. Rev. Lett. 112 (2014) 082001, [arXiv:1310.7192].

[13] M. Dasgupta, F. A. Dreyer, G. P. Salam, and G. Soyez, Inclusive jet spectrum for small-radius jets, JHEP 06 (2016) 057, [arXiv: 1602 .01110].

[14] Z.-B. Kang, F. Ringer, and I. Vitev, The semi-inclusive jet function in SCET and small radius resummation for inclusive jet production, JHEP 10 (2016) 125, [arXiv:1606.06732].

[15] T. Becher, M. Neubert, L. Rothen, and D. Y. Shao, Effective Field Theory for Jet Processes, Phys. Rev. Lett. 116 (2016), no. 19 192001, [arXiv: 1508.06645 ].

[16] X. Liu, S.-O. Moch, and F. Ringer, Phenomenology of single-inclusive jet production with jet radius and threshold resummation, Phys. Rev. D97 (2018), no. 5 056026, [arXiv: 1801.07284 ].

[17] Z. Nagy and D. E. Soper, Jets and threshold summation in Deductor, Phys. Rev. D98 (2018), no. 1 014035, [arXiv:1711.02369].

[18] S. Dulat, T.-J. Hou, J. Gao, M. Guzzi, J. Huston, P. Nadolsky, J. Pumplin, C. Schmidt, D. Stump, and C. P. Yuan, New parton distribution functions from a global analysis of quantum chromodynamics, Phys. Rev. D93 (2016), no. 3 033006, [arXiv: 1506.07443 ].

[19] CMS Collaboration, V. Khachatryan et al., Measurement and QCD analysis of double-differential inclusive jet cross sections in pp collisions at $\sqrt{S}=8 \mathrm{TeV}$ and cross section ratios to 2.76 and $7 \mathrm{TeV}$, JHEP 03 (2017) 156, [arXiv: 1609.05331$].$

[20] fastNLO Collaboration, D. Britzger, K. Rabbertz, F. Stober, and M. Wobisch, New features in version 2 of the fastNLO project, in Proceedings, 20th International Workshop on Deep-Inelastic Scattering and Related Subjects (DIS 2012): Bonn, Germany, March 26-30, 2012, pp. 217-221, 2012. arXiv:1208.3641. 
[21] M. Cacciari, G. P. Salam, and G. Soyez, The anti-k $k_{t}$ jet clustering algorithm, JHEP 04 (2008) 063, [arXiv:0802.1189].

[22] E. Eren, Determination of the Parton Distribution Functions and Strong Coupling by Using Measurement of Jet Cross Sections at $\sqrt{S}=13 \mathrm{TeV}$ with the CMS detector with a combined QCD Analysis, Hamburg University, Ph.D. thesis, 2018.

[23] S. Alekhin et al., HERAFitter, Eur. Phys. J. C75 (2015), no. 7 304, [arXiv: 1410.4412 ].

[24] xFitter Developers' Team Collaboration, V. Bertone et al., xFitter 2.0.0: An Open Source QCD Fit Framework, PoS DIS2017 (2018) 203, [arXiv:1709.01151]. 